\title{
The Future of Parkinson Care and Research is Here
}

\author{
Bastiaan R. Bloem ${ }^{\mathrm{a}, *}$ and Patrik Brundin ${ }^{\mathrm{b}}$ \\ ${ }^{a}$ Radboud University Nijmegen Medical Center; Donders Institute for Brain, Cognition and Behavior; Center \\ of Expertise for Parkinson \& Movement Disorders; Department of Neurology; Nijmegen, the Netherlands \\ ${ }^{\mathrm{b}}$ Center for Neurodegenerative Science, Van Andel Research Institute, Grand Rapids, MI, USA
}

Keywords: Parkinson's disease, research, clinical care, innovation

The field of Parkinson's disease research is increasingly dynamic. These are promising times for affected patients and their families, with improved healthcare services being in practice already, and with new potentially disease-modifying treatments on the horizon. It is also a gratifying time for clinicians, who now can provide more hope when conveying the diagnosis to patients, and who can offer them new evidence-based treatments. And these are certainly also exciting times for scientists, who can capitalise on new technologies which are providing fast-rising insights in fundamental disease mechanisms, who can develop innovative interventions tailored to these novel concepts, and build a robust evidence base for them. This new, rich and versatile issue of the Journal of Parkinson's Disease is a good illustration of these many promising developments in the field, and we hope you will enjoy reading as much we did.

Meanwhile, the journal itself is taking a big step forward. One important new development for this coming year is the arrival of Bas Bloem as the new co-Editor-in-Chief, who will replace Bill Langston

\footnotetext{
${ }^{*}$ Correspondence to: Prof. Bastiaan R. Bloem, MD, PhD, FRCPE, Department of Neurology (935), Radboud University Nijmegen Medical Center, PO Box 9101, 6500 HB Nijmegen, The Netherlands. Tel.: +31 243615 202; E-mail: bas.bloem@rad boudumc.nl.
}

as sparring partner for Patrik Brundin, who will continue in his role as co-Editor-in-Chief. We would like to take this opportunity to thank Bill for his tremendous contribution to the Journal of Parkinson's Disease, as one the two founding fathers. At the outset, Bill instantly grasped the need for a new journal, catering as it does for high-quality research in Parkinson's disease across the spectrum of basic and clinically oriented work. Together with Patrik, he helped design the Editorial Board and was vital in securing the support of many key scientists in the field to join and support the journal, which was a critical factor in ascertaining its early successes. Over the first 10 years of the journal, Bill's input and gravitas have been invaluable. Not only has he been a coarchitect of three special issues, he also helped steer the journal strategically at several crucial junctures. It is no exaggeration to say that the Journal of Parkinson's Disease would not stand where it does today without him.

We also want to take this opportunity to highlight just a few of Bill's seminal contributions to the field of Parkinson's at large. For many years, he has been a thought leader in the field. For example, he has played a key role as a strategic scientific advisor for several funding agencies, and he was quick to recognise and communicate that 
Parkinson's disease is much more than a movement disorder [1]. His scientific journey into Parkinson's disease started with his classic observations [2-4] on the severe and irreversible parkinsonism that developed in young adults who had injected themselves with a 'new synthetic heroin', which inadvertently was contaminated with 1-methyl-4phenyl-1,2,3,6-tetrahydropyridine (MPTP). These observations completely changed the landscape of Parkinson's research. An important impact was that it led to the development of new cell and animal models for basic research, and it put focus on a possible role of mitochondria in Parkinson's long before there was genetic evidence that they might play a key role in the pathogenesis. One of several other important consequences of this discovery was a surge of interest into the possible role of environmental toxins, in large part due to the chemical and neurotoxic similarities between MPTP and widely used pesticides such as paraquat and rotenone [5] This environmental hypothesis is now more alive than ever, with a true pandemic Parkinson happening around us today, which might be linked in part to exposure to pesticides and other environmental toxins [6]. Bill also swiftly took his clinical observations into the laboratory, showing that the toxic effects of MPTP in animals could be countered by MAO-B inhibitors. This in turn led to the first attempts to pharmacologically slow down the clinical course of Parkinson's disease, [7] which - albeit in hindsight unsuccessful - motivated next generations to further pursue the holy grail of disease modification.

In 1989, the MPTP patients that Bill had identified a few years earlier came to affect the Parkinson research field once again. Two of these patients, and eventually one additional case, received intracerebral transplants of fetal dopamine neurons in Sweden, and these implants were later reported to mitigate some of their motor symptoms [8]. The notion was that this was the ultimate paradigm to test the clinical potential of dopamine neuron transplants since - unlike the situation in idiopathic Parkinson's disease - it was possible to guarantee that the disease-causing trigger (MPTP) was no longer present in these patients. As it happens, one of the members of the Swedish clinical transplant team was the, then young, post-doc Patrik Brundin. Already 30 years ago, Patrik and Bill forged a friendship that helped them to successfully launch the Journal in 2010.

During his career, Bill has also continued to mentor many other young people on their road to become independent scientists or clinicians in the field of
Parkinson's disease. Incidentally, one of them was a young medical student named Bas Bloem, who is now his successor here for the journal. Their collaboration also started in the late eighties, with work on the role of the dopaminergic system in controlling postural reflexes, again using the persons with MPTP as an ideal test model $[9,10]$. The friendship and alliance between Bill and Bas continues until today, with a recent publication that analyses the original video footage of the MPTP patients [11]. Specifically, careful retrospective inspection showed a complete absence of freezing of gait when these patients initially presented in a severely affected yet still untreated state, although freezing did develop months after levodopa treatment was started, suggesting that dopaminergic treatment may play a causative role in the development of freezing [11].

It gives us great pleasure to announce that Bill will not leave the journal behind, but will stay on board as Editor-in-Chief Emeritus, so we can keep counting on his senior advice for years to come, as we move collectively into the future of Parkinson's care and research.

\section{CONFLICTS OF INTEREST}

Other than their personal friendship with Bill Langston, BRB and PB have no conflicts of interest that are relevant to this publication.

\section{ACKNOWLEDGEMENTS}

BRB was supported by a center of excellence grant of the Parkinson's Foundation.

\section{REFERENCES}

[1] Langston JW (2006) The Parkinson's complex: Parkinsonism is just the tip of the iceberg. Ann Neurol 59, 591-596.

[2] Langston JW, Ballard JW, Tetrud JW, Irwin I (1983) Chronic parkinsonism in humans due to a product of meperidine-analog synthesis. Science 219, 979-980.

[3] Ballard PA, Tetrud JW, Langston JW (1985) Permanent human parkinsonism due to 1-methyl-4-phenyl-1,2,3,6tetrahydropyridine (MPTP): Seven cases. Neurology 35, 949-956.

[4] Langston JW (2017) The MPTP Story. J Parkinsons Dis 7(s1), S11-s9.

[5] Tanner CM, Kamel F, Ross GW, et al. (2011) Rotenone, paraquat, and Parkinson's disease. Environ Health Perspect 119(6), 866-872.

[6] Dorsey ER, Sherer T, Okun MS, Bloem BR (2018) The Emerging Evidence of the Parkinson Pandemic. J Parkinsons Dis 8(s1), S3-s8. 
[7] Parkinson Study Group (1989) Effect of deprenyl on the progression of disability in early Parkinson's disease. The Parkinson Study Group. N Engl J Med 321, 1364-1371.

[8] Widner H, Tetrud J, Rehncrona S, et al. (1992) Bilateral fetal mesencephalic grafting in two patients with parkinsonism induced by 1-methyl-4-phenyl-1,2,3,6-tetrahydropyridine (MPTP). N Engl J Med 327(22), 1556-1563.

[9] Bloem BR, Irwin I, Haan J, et al. (1990) The MPTP model: Versatile contributions to the treatment of idiopathic Parkinson's disease. J Neurol Sci 97, 273-293.
[10] Bloem BR, Beckley DJ, van Dijk JG, et al. (1994) Medium latency stretch reflexes in young-onset Parkinson's disease and MPTP-induced parkinsonism. J Neurol Sci 123, 52-58.

[11] Nonnekes J, Post B, Tetrud JW, Langston JW, Bloem BR (2018) MPTP-induced parkinsonism: An historical case series. Lancet Neurol 17(4), 300-301. 